\title{
Raw material variability as archaeological tools: Preliminary results from a geochemical study of the basalt vessel workshop at Iron Age Tel Hazor, Israel
}

\author{
Tatjana Gluhak ${ }^{1}$, Danny Rosenberg ${ }^{2}$, Jennie Ebeling ${ }^{3}$ \\ 1. Institute of Geosciences, Johannes Gutenberg University, Mainz, Germany. Email: gluhak@uni-mainz.de \\ 2. Laboratory for Ground Stone Tools Research, Zinman Institute of Archaeology, University of Haifa, Haifa, \\ Israel. Email: drosenberg@research.haifa.ac.il \\ 3. Department of Archaeology and Art History, University of Evansville, Evansville, IN, U.S.A. \\ Email: je55@evansville.edu
}

\begin{abstract}
:
The discovery of a basalt vessel workshop at Tel Hazor, one of the most important Iron Age sites in the Near East, marks a turning point in our understanding of stone artefact production and distribution during the $1^{\text {st }}$ millennium BCE. It offers a rare opportunity to characterize ancient raw material sources, production sites, and study production, trade and distribution systems. The basalt vessel workshop, the only one of its kind in the Levant, produced large quantities of bowl preforms and production waste. To better understand the production and distribution systems behind this specialized production centre, in 2011 we initiated a focused geochemical project that concentrated on the products of this unique workshop. We measured the major and trace element composition of 44 unfinished basalt vessels from the workshop and other contexts at Hazor, and can demonstrate that the majority of these objects were derived from one specific, geochemically well-constrained, basaltic rock source. Only a few bowls clearly deviate from this geochemical composition and were produced using raw material from other sources. Thus, we believe that one major quarry existed that supplied the Hazor workshop with the majority of the basaltic raw material. The products from this specific extraction site provide us with a "Hazor reference group" that can be used to test whether or not finished vessels from Hazor and contemporary sites were produced in the Hazor workshop.
\end{abstract}

Keywords: ground stone tools; basalt vessels; workshop; geochemistry; Iron Age; Tel Hazor; Israel

\section{Introduction}

Finely-carved basalt vessels were both functional tools and status objects in the Iron Age (ca. 1200-586 BCE) southern Levant and their ubiquity in archaeological contexts throughout the region, including areas lacking basalt deposits, demonstrates their value to the Iron Age population. Although many goods, including textiles, spices, oils, and metals, were acquired over long distances during the Iron Age, few are amenable to provenance study. Stone artefacts, on the other hand, "have a far greater potential for elucidating long distance trade

Published by the School of History, Classics and Archaeology, University of Edinburgh ISSN: 2055-0472. URL: http://journals.ed.ac.uk/lithicstudies/

This work is licensed under a Creative Commons Attribution 2.5 UK: Scotland License. 
networks, as they are relatively common, virtually indestructible and do not generally undergo chemical or physical changes during their manufacture, use or subsequent deposition" (Rutter et al. 2003: 209). The recent discovery of a workshop for the manufacture of basalt vessels in a $9^{\text {th }}$ century BCE context at Hazor (Ebeling \& Rosenberg 2015) provides a unique opportunity to investigate aspects of the manufacture and distribution of this well-known but little-understood aspect of Israelite material culture.

Stone vessels found in Egypt and the eastern Mediterranean world have been the focus of archaeological interest during the past two decades. Stocks' studies $(1993 ; 1999 ; 2003)$ of the manufacture of hard stone objects, including vessels, sarcophagi, and statues, in ancient Egypt using archaeological, ethnographic, and experimental approaches, have shed much light on our understanding of manufacturing methods, especially the manufacture of vessels made of hard stone like basalt. Bevan (2007: 1), in his study of stone vessels and values in the Mediterranean Bronze Age, states that stone vessels are desirable sources of study for, among other reasons, their virtual indestructibility, the subtractive properties of stone, and the potential for macroscopic, petrographic, and geochemical provenance study. In her study of $2^{\text {nd }}$ millennium BCE stone vessels in the Levant, Sparks (2007: 179-183) describes the evidence for the manufacture of obsidian and granite vessels at Middle Bronze Age Atchana (Alalakh) in southern Turkey and the manufacture of gypsum vessels at Middle Bronze IILate Bronze Age Bet Shean in Israel.

A number of provenance studies have been conducted on basalt artefacts dated to different periods in the region since the 1980s, but these past attempts produced rather limited results and were only able to constrain larger regions (e.g., Amiran \& Porat 1984; Philip \& Williams-Thorpe 1993; 2000; 2001; Williams-Thorpe \& Thorpe 1993; Rowan 1998; Rutter 2003; Rutter et al. 2003: 210; Watts et al. 2004; Rutter \& Philip 2008). Petrographic studies either did not include detailed geochemical analyses (Amiran \& Porat 1984) or focused solely on the artefacts without an intensive geochemical study of the potential basaltic raw material in the field. In their analyses, Philip \& Williams-Thorpe, who only included 21 geological samples from Jordan (Philip \& Williams-Thorpe 1993) and later added eight geological field samples (Philip \& Williams-Thorpe 2001), stressed that more exact provenance determinations would only be possible with a more elaborate study of the basaltic rocks themselves and the establishment of a source database that facilitates statistical evaluations (Philip \& Williams-Thorpe 2001).

The studies of Rutter (2003), Rutter \& Philip (2008), and Rutter et al. (2003) concentrated on artefacts as well as the evaluation of trace element analyses; however, they left out basic analyses of rock types as well as an elaborate evaluation of their major element compositions. Furthermore, they based their constriction of the volcanic rocks in the region on a very small number of geological field samples in addition to those collected by Philip and Williams-Thorpe (1993; 2001). However, these authors, especially Rutter et al. (2003), stress that more geological samples must be analysed to gain a clearer picture of artefact provenance. An intensive study of the raw material variability of the artefacts connected to statistical evaluations was not conducted in any of the studies. Geochemical analyses undertaken in the 1980s led Xenophontos et al. (1988: 181) to speculate that Hazor may have been a centre for the trade in basalt vessels to Cyprus in the Late Bronze Age and Roman periods, although the authors of this study could not distinguish between the Syrian, Palestinian, and Jordanian basalt sources.

The geochemical analyses conducted by Rutter (2003) using ICP-MS (inductively coupled plasma mass spectrometry) suggest that ground stone artefacts found at Hazor were carved from basalt sources in the north Jordan Valley, Golan Heights, and Mt. Hermon regions (Rutter 2003: table 8:11). However, as stated above, these studies reflect the potential of provenance studies while also demonstrating the problems inherent in these studies, in 
particular the need for a reliable geochemical database of basalt sources. A large-scale project was recently initiated that focuses on establishing the geochemical signature of basalt sources in Israel using a basanite bifacial tool quarry and production site in the Manasseh Hills at the western fringes of the Jezreel Valley as a test case for the chosen methodology (Gluhak \& Rosenberg 2013; Rosenberg \& Gluhak 2016). The results of the study proved that a highresolution sampling strategy, the geochemical analyses chosen, and statistical, multivariate analyses (see methodology section, below), provide a good combination for studying the provenance of basaltic tools. This was the springboard for the current study.

In 2010, the first workshop for the production of basalt vessels identified in the Levant was discovered at Hazor in northern Galilee. The largest Bronze and Iron Age tell site in northern Israel, Yadin excavated Hazor between 1955-1958 and again in 1968; the current excavation project directed by Ben-Tor commenced in 1990 (Ben-Tor 1989; Ben-Tor \& Bonfil 1997; Ben-Tor \& Zuckerman 2010). During 25 excavation seasons, thousands of ground stone tools, vessels, and other stone artefacts have been excavated from Bronze Age, Iron Age, and Persian Period contexts (Ebeling 2012). Located on the northern edge of Tel Hazor, the workshop was identified in a space defined by walls to the north and east with baulks up to three metres high to the west and south.

Two dozen blanks and unfinished basalt vessels of several types were associated with the workshop and the floors and fills contained flint tools, basalt hammerstones, and basalt production waste, as well as two iron chisels, a silver earring, spindle whorls, shells, and a zoomorphic figurine (Ebeling \& Rosenberg 2015). In addition, 25 unfinished basalt vessels excavated between the 1990 and 2007 seasons in various contexts on the tell were also identified during analysis of the large ground-stone assemblage; at least two, although probably several others as well, were found in Late Bronze Age contexts, suggesting that an earlier basalt vessel industry also existed at the site. Forty-four samples from the total assemblage of 49 vessels were selected for geochemical analysis.

Geologically, Tel Hazor is located in the southwestern part of the Hula Valley in close vicinity to basaltic rocks that could have served as raw material for vessel production. The Hula Valley is a pull-apart basin along the Dead Sea transform filled with lacustrine and clastic sediments reaching a thickness of up to $3.5 \mathrm{~km}$ (e.g., Schattner \& Weinberger 2008). Plio- to Pleistocene volcanic units are intercalated in the basin fill that can be stratigraphically connected to the Hasbani basalt in the north, the Golan volcanic rocks to the east, the Korazin block to the south, or the Dalton basalt in the southwest. In the immediate surroundings of Tel Hazor, where creeks cut the basin sediments, small outcrops of Pliocene Dalton basalt occur, whereas further to the east and southeast of Tel Hazor, the basaltic rocks cropping out within the basin sediments can be linked to the Ruman Yarda flows of the Golan volcanic massif that borders the Hula Basin in the east (see Figure 1) (Sneh \& Weinberger 2006).

The purpose of the current geochemical-mineralogical analyses of the basalt vessels is to determine how many different basaltic raw materials sources were used by the craftspeople of Tel Hazor. The raw material variability within the present group of 44 basalt vessels offers important information about basaltic rock procurement as we can determine if the workshop was supplied with raw material from one or more specific basaltic rock extraction sites. This could suggest a close connection between extraction site and craftsperson, a more widespread procurement system, or even the absence of a well-defined procurement system for basaltic rock raw material. However, if within the group of basaltic vessels a specific rock can be identified geochemically as a dominant raw material used for vessel production at Tel Hazor, one or more "Tel Hazor reference groups" could be defined. These geochemical "reference groups" could serve in future basalt vessel provenance analyses as a basis to check whether or not a vessel found elsewhere was produced at Tel Hazor. Furthermore, assuming that one or more dominant extraction sites existed, the geochemical-mineralogical data of the artefacts 
serve as the basis to track their provenance when compared to geological samples collected in the field.

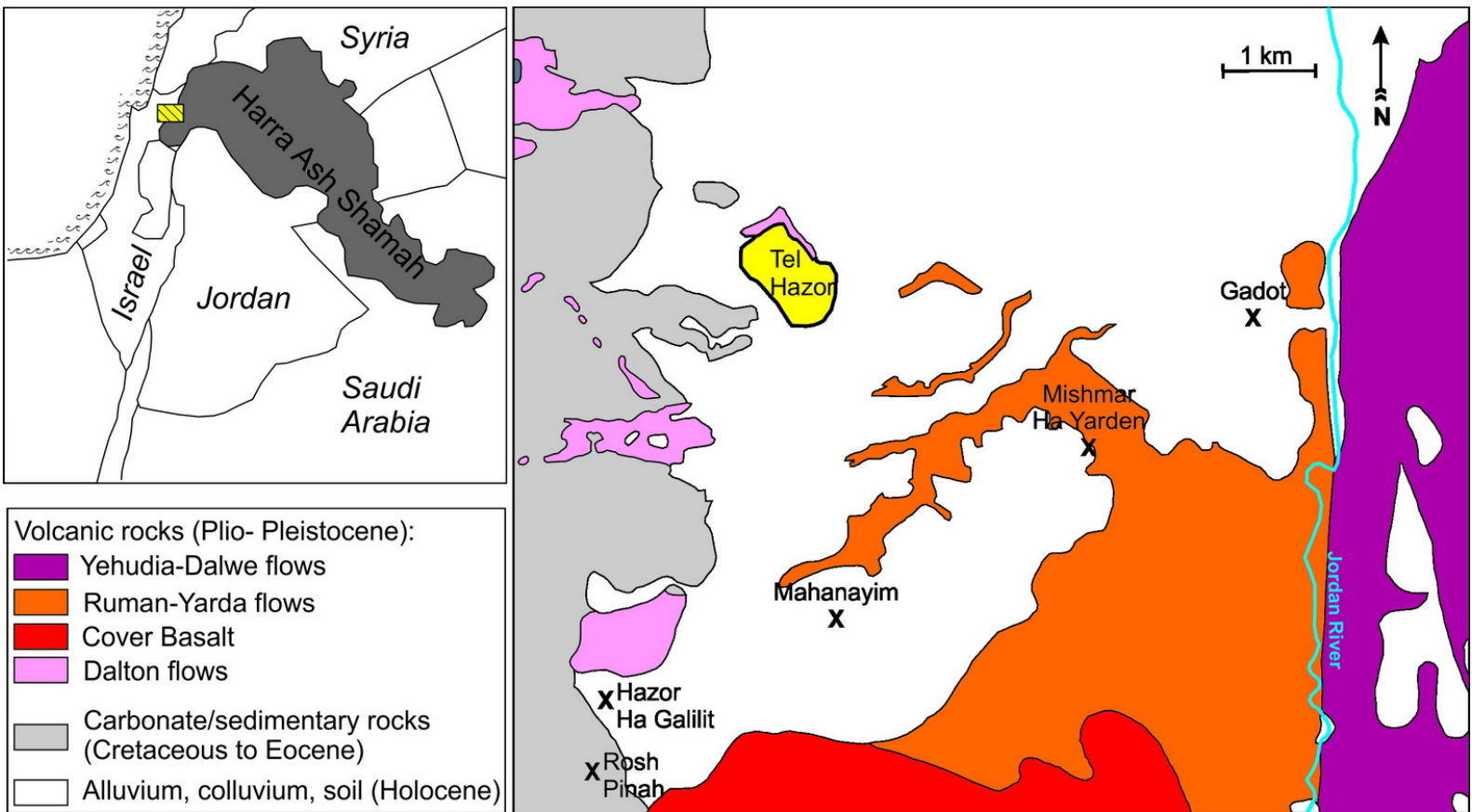

Figure 1: The geological situation of Tel Hazor in the southern Hula basin. Detail of the Rosh Pinah geological map (Sneh \& Weinberger 2006).

\section{Materials and methods}

The vessels are produced from grey lava with up to 5\% unevenly distributed vesicles in sub-mm to $\mathrm{mm}$ size. The only macroscopically visible phenocrysts are olivines, strongly altered to iddingsite. For the geochemical mineralogical analyses, a small piece was chipped off each of the 44 basaltic vessels. The sampling was conducted at the Laboratory for Groundstone Tools Research at the Zinman Institute of Archaeology at the University of Haifa. The samples were then sent to the Institute for Geosciences of the Johannes GutenbergUniversity in Mainz. Seven samples were large enough to enable the production of thin sections for petrography. Weathered or carbonate crust was either removed by abrasion on a grinding wheel or by rinsing in $\mathrm{HCl}$. Afterward, the samples were rinsed in distilled water in an ultrasonic bath, dried, and ground to powder manually in an agate mortar. As only a very limited amount of sample material was available and to avoid any contamination, the loss on ignition was determined in a platinum-cup on about 0.5-1.0 g of sample powder.

The same "ignited" material was used afterward for the geochemical analyses. To produce glass beads for major element determination by X-ray fluorescence, $0.4 \mathrm{~g}$ of ignited sample powder was fused with $5.2 \mathrm{~g}$ of $\mathrm{Li}_{2} \mathrm{~B}_{4} \mathrm{O}_{7}$. The major element analyses were conducted in a 2002 model Philips MagXPRO wavelength-dispersive X-ray spectrometer with a Rh-Xray tube and a maximum excitation of $3.2 \mathrm{~kW}$. For trace element determination by LaserAblation-ICP-MS, approximately $40 \mathrm{mg}$ of the sample powder was melted at $1200{ }^{\circ} \mathrm{C}$ to a glass bead in an iridium strip-heater. Three spots with a diameter of $100 \mu \mathrm{m}$ each were measured on every glass bead using a pulse-rate of $10 \mathrm{~Hz}$ and laser energy densities of 6 $\mathrm{J} / \mathrm{cm}^{2}$ in an Agilent $7500 \mathrm{CE}$ quadrupol ICP-MS, coupled to an ESI New Wave Research NWR193 (ArF-excimer) laser-ablation-system with $193 \mathrm{~nm}$ wavelength. The carrier gas was Argon. ${ }^{43} \mathrm{Ca}$ served as the internal standard, its values taken from the XRF measurements. The reference materials NIST SRM 610 and NIST SRM 612 served as the external standards. The USGS-BCR-2G was measured for quality control. The values for NIST SRM 610, NIST 
SRM 612, and USGS-BCR-2G were taken from the GeoRem Database (GeoRem preferred values, Jochum et al. 2005). Reproducibility and accuracy of the LA-ICP-MS analyses, monitored by repeated measurements of USGS-BCR-2G, are presented in Table 1. Data reduction and evaluation was conducted in MS excel. Results are presented in Table 2.

Table 1: Reproducibility and accuracy of the LA-ICP-MS analyses. Abbreviations: SD - standard deviation, RSD - relative standard deviation. GEOREM preferred values from Jochum et al. (2005).

\begin{tabular}{|c|c|c|c|c|}
\hline & \multicolumn{4}{|c|}{ USGS-BCR-2G } \\
\hline & average $(n=42)$ & SD & RSD \% & $\begin{array}{c}\text { GEOREM } \\
\text { preferred values }\end{array}$ \\
\hline Sc & 36.23 & 1.75 & 4.84 & 33 \\
\hline $\mathbf{V}$ & 440.80 & 14.05 & 3.19 & 425 \\
\hline $\mathrm{Cr}$ & 15.40 & 1.32 & 8.55 & 17 \\
\hline$M n$ & 1611.47 & 73.94 & 4.59 & 1550 \\
\hline Co & 38.10 & 1.61 & 4.23 & 38 \\
\hline $\mathbf{N i}$ & 12.32 & 0.89 & 7.20 & 13 \\
\hline Ga & 21.74 & 0.96 & 4.42 & 23 \\
\hline $\mathbf{R b}$ & 47.49 & 2.57 & 5.41 & 47 \\
\hline $\mathrm{Sr}$ & 330.43 & 9.32 & 2.82 & 342 \\
\hline $\mathbf{Y}$ & 33.33 & 1.18 & 3.55 & 35 \\
\hline $\mathrm{Zr}$ & 182.03 & 5.71 & 3.14 & 184 \\
\hline $\mathrm{Nb}$ & 12.68 & 0.38 & 3.02 & 12.5 \\
\hline $\mathrm{Ba}$ & 670.97 & 33.78 & 5.03 & 683 \\
\hline La & 24.52 & 0.98 & 4.00 & 24.7 \\
\hline $\mathrm{Ce}$ & 51.05 & 1.84 & 3.60 & 53.3 \\
\hline $\operatorname{Pr}$ & 6.38 & 0.22 & 3.45 & 6.7 \\
\hline Nd & 28.40 & 1.19 & 4.19 & 28.9 \\
\hline Sm & 6.47 & 0.43 & 6.58 & 6.59 \\
\hline Eu & 1.94 & 0.10 & 5.20 & 1.97 \\
\hline Gd & 6.48 & 0.41 & 6.29 & 6.71 \\
\hline Tb & 0.96 & 0.06 & 6.44 & 1.02 \\
\hline Dy & 6.19 & 0.34 & 5.46 & 6.44 \\
\hline Ho & 1.23 & 0.08 & 6.57 & 1.27 \\
\hline Er & 3.64 & 0.21 & 5.82 & 3.70 \\
\hline Tm & 0.48 & 0.04 & 8.08 & 0.51 \\
\hline $\mathrm{Yb}$ & 3.28 & 0.22 & 6.79 & 3.39 \\
\hline Lu & 0.48 & 0.04 & 8.94 & 0.50 \\
\hline $\mathrm{Hf}$ & 4.87 & 0.26 & 5.37 & 4.84 \\
\hline Ta & 0.73 & 0.04 & 6.01 & 0.78 \\
\hline Th & 5.88 & 0.28 & 4.75 & 5.90 \\
\hline $\mathbf{U}$ & 1.68 & 0.09 & 5.47 & 1.69 \\
\hline
\end{tabular}


Table 2. Major and trace element composition of the Tel Hazor basalt vessels. Abbreviations: RMS - root mean square.

\begin{tabular}{|c|c|c|c|c|c|c|c|c|c|c|c|c|c|c|c|}
\hline & Haz1 & Haz2 & Haz3 & Haz4 & Haz5 & Haz6 & Haz7 & Haz8 & Haz9 & Haz10 & Haz11 & Haz12 & Haz13 & Haz14 & Haz15 \\
\hline $\mathrm{SiO}_{2}(\%)$ & 48.42 & 48.95 & 48.77 & 49.01 & 48.89 & 49.00 & 47.95 & 48.37 & 48.97 & 48.22 & 48.80 & 48.83 & 48.70 & 48.06 & 48.89 \\
\hline $\mathrm{Al}_{2} \mathrm{O}_{3}$ & 15.01 & 15.94 & 15.26 & 15.76 & 15.50 & 15.62 & 15.35 & 15.08 & 15.51 & 15.70 & 15.81 & 15.62 & 15.57 & 15.32 & 15.43 \\
\hline $\mathrm{Fe}_{2} \mathrm{O}_{3}(\mathrm{t})$ & 13.42 & 12.62 & 12.97 & 12.67 & 12.72 & 12.88 & 12.73 & 13.10 & 12.92 & 12.18 & 12.96 & 12.80 & 12.78 & 12.43 & 12.96 \\
\hline $\mathrm{MgO}$ & 7.26 & 6.30 & 7.24 & 6.42 & 6.70 & 6.72 & 6.65 & 7.55 & 6.25 & 6.04 & 6.73 & 6.78 & 6.79 & 6.61 & 7.05 \\
\hline $\mathrm{CaO}$ & 9.42 & 9.74 & 9.39 & 9.95 & 9.77 & 9.65 & 10.90 & 9.23 & 9.84 & 11.60 & 9.29 & 9.30 & 9.60 & 11.41 & 9.56 \\
\hline $\mathrm{Na}_{2} \mathrm{O}$ & 3.07 & 3.14 & 3.02 & 3.15 & 3.13 & 3.14 & 3.04 & 3.02 & 2.96 & 3.11 & 3.10 & 3.13 & 3.12 & 3.10 & 3.12 \\
\hline $\mathrm{K}_{2} \mathrm{O}$ & 0.94 & 0.83 & 0.88 & 0.89 & 0.91 & 0.88 & 0.85 & 0.81 & 0.88 & 0.83 & 0.87 & 0.90 & 0.87 & 0.89 & 0.84 \\
\hline $\mathrm{TiO}_{2}$ & 2.16 & 2.00 & 2.05 & 2.09 & 2.11 & 2.05 & 2.05 & 2.03 & 2.16 & 1.94 & 2.06 & 2.04 & 1.99 & 2.01 & 2.07 \\
\hline $\mathrm{P}_{2} \mathrm{O}_{5}$ & 0.50 & 0.40 & 0.44 & 0.44 & 0.44 & 0.45 & 0.44 & 0.38 & 0.42 & 0.42 & 0.45 & 0.45 & 0.38 & 0.45 & 0.40 \\
\hline sum & 100.20 & 99.92 & 100.02 & 100.38 & 100.17 & 100.39 & 99.96 & 99.57 & 99.91 & 100.04 & 100.07 & 99.85 & 99.80 & 100.28 & 100.32 \\
\hline LOI & 0.52 & 0.05 & 0.09 & 0.56 & 0.00 & 0.00 & 1.35 & 0.00 & 0.52 & 1.42 & 0.17 & 0.13 & 0.17 & 1.58 & 0.00 \\
\hline Sc (ppm) & 26 & 24 & 27 & 27 & 27 & 26 & 25 & 25 & 27 & 25 & 31 & 27 & 26 & 25 & 27 \\
\hline $\mathrm{V}$ & 223 & 195 & 231 & 214 & 205 & 233 & 214 & 220 & 231 & 205 & 206 & 219 & 218 & 210 & 219 \\
\hline $\mathrm{Cr}$ & 266 & 183 & 347 & 292 & 223 & 251 & 240 & 250 & 276 & 267 & 220 & 244 & 254 & 305 & 247 \\
\hline $\mathrm{Mn}$ & 1327 & 1221 & 1448 & 1426 & 1264 & 1311 & 1331 & 1433 & 1243 & 1233 & 1288 & 1311 & 1265 & 1313 & 1334 \\
\hline Co & 48 & 40 & 51 & 47 & 43 & 47 & 46 & 51 & 39 & 42 & 48 & 45 & 47 & 45 & 49 \\
\hline $\mathrm{Ni}$ & 135 & 68 & 135 & 105 & 94 & 110 & 109 & 148 & 76 & 77 & 116 & 113 & 130 & 106 & 133 \\
\hline $\mathrm{Ga}$ & 21 & 21 & 22 & 22 & 21 & 21 & 21 & 22 & 21 & 20 & 23 & 22 & 21 & 22 & 22 \\
\hline $\mathrm{Rb}$ & 12 & 13 & 12 & 13 & 12 & 10 & 12 & 12 & 12 & 11 & 13 & 13 & 13 & 12 & 12 \\
\hline $\mathrm{Sr}$ & 555 & 533 & 570 & 585 & 588 & 589 & 579 & 484 & 493 & 530 & 598 & 591 & 503 & 578 & 523 \\
\hline$Y$ & 23 & 20 & 21 & 38 & 20 & 20 & 19 & 20 & 22 & 19 & 23 & 23 & 19 & 27 & 22 \\
\hline $\mathrm{Zr}$ & 170 & 145 & 164 & 155 & 148 & 152 & 148 & 139 & 157 & 143 & 176 & 156 & 142 & 148 & 163 \\
\hline $\mathrm{Nb}$ & 30.9 & 31.3 & 24.7 & 27.5 & 25.9 & 26.1 & 25.9 & 24.2 & 34.9 & 30.6 & 33.3 & 26.8 & 24.9 & 27.2 & 29.4 \\
\hline $\mathrm{Ba}$ & 299 & 262 & 295 & 319 & 281 & 282 & 296 & 247 & 237 & 294 & 300 & 318 & 261 & 284 & 259 \\
\hline La & 26.5 & 20.8 & 24.8 & 24.4 & 24.3 & 24.2 & 23.0 & 20.3 & 22.4 & 20.3 & 26.7 & 27.4 & 19.8 & 24.2 & 23.7 \\
\hline $\mathrm{Ce}$ & 51.7 & 40.8 & 50.8 & 49.6 & 48.0 & 48.5 & 46.2 & 42.4 & 43.0 & 42.7 & 50.3 & 54.3 & 39.9 & 49.1 & 45.9 \\
\hline $\mathrm{Pr}$ & 6.4 & 5.2 & 6.1 & 5.9 & 5.8 & 5.7 & 5.6 & 5.1 & 5.7 & 5.1 & 6.2 & 6.5 & 4.8 & 5.8 & 5.6 \\
\hline
\end{tabular}




\begin{tabular}{|c|c|c|c|c|c|c|c|c|c|c|c|c|c|c|c|}
\hline & Haz1 & Haz2 & Haz3 & Haz4 & Haz5 & Haz6 & Haz7 & Haz8 & Haz9 & Haz10 & Haz11 & Haz12 & Haz13 & Haz14 & Haz15 \\
\hline $\mathrm{Nd}$ & 27.0 & 22.0 & 25.7 & 25.0 & 25.1 & 24.1 & 24.0 & 21.7 & 24.7 & 22.3 & 27.2 & 28.1 & 21.4 & 24.6 & 24.7 \\
\hline Sm & 5.85 & 5.05 & 5.22 & 5.60 & 5.70 & 5.41 & 4.92 & 5.03 & 5.39 & 5.02 & 6.11 & 5.90 & 4.84 & 5.37 & 5.50 \\
\hline $\mathrm{Eu}$ & 1.92 & 1.70 & 1.95 & 2.00 & 1.85 & 1.82 & 1.79 & 1.69 & 1.85 & 1.84 & 1.95 & 2.01 & 1.66 & 1.88 & 2.03 \\
\hline $\mathrm{Gd}$ & 5.39 & 4.71 & 5.34 & 5.01 & 4.74 & 5.26 & 4.77 & 4.39 & 5.30 & 4.52 & 5.85 & 5.89 & 4.41 & 4.50 & 5.66 \\
\hline $\mathrm{Tb}$ & 0.79 & 0.69 & 0.73 & 0.69 & 0.73 & 0.71 & 0.71 & 0.67 & 0.75 & 0.66 & 0.77 & 0.81 & 0.68 & 0.70 & 0.80 \\
\hline Dy & 4.69 & 3.86 & 4.14 & 4.17 & 4.08 & 4.12 & 4.00 & 3.97 & 4.52 & 3.95 & 4.77 & 4.87 & 3.94 & 4.04 & 4.86 \\
\hline Ho & 0.81 & 0.73 & 0.83 & 0.76 & 0.80 & 0.70 & 0.70 & 0.75 & 0.85 & 0.72 & 0.89 & 0.85 & 0.72 & 0.73 & 0.87 \\
\hline $\mathrm{Er}$ & 2.20 & 1.96 & 2.00 & 2.07 & 2.26 & 1.94 & 1.92 & 1.99 & 2.19 & 1.97 & 2.31 & 2.42 & 1.92 & 1.90 & 2.20 \\
\hline Tm & 0.27 & 0.24 & 0.21 & 0.26 & 0.23 & 0.25 & 0.21 & 0.24 & 0.29 & 0.24 & 0.27 & 0.24 & 0.28 & 0.23 & 0.26 \\
\hline $\mathrm{Yb}$ & 1.82 & 1.60 & 1.73 & 1.64 & 1.49 & 1.80 & 1.48 & 1.54 & 1.88 & 1.36 & 1.84 & 1.88 & 1.53 & 1.64 & 1.99 \\
\hline Lu & 0.24 & 0.21 & 0.23 & 0.23 & 0.24 & 0.21 & 0.21 & 0.22 & 0.27 & 0.21 & 0.25 & 0.25 & 0.21 & 0.22 & 0.21 \\
\hline $\mathrm{Hf}$ & 3.70 & 3.32 & 3.55 & 3.51 & 3.45 & 3.23 & 3.32 & 3.30 & 3.45 & 3.13 & 3.90 & 3.69 & 3.48 & 3.16 & 3.78 \\
\hline Ta & 1.57 & 1.58 & 1.18 & 1.27 & 1.30 & 1.23 & 1.37 & 1.16 & 1.72 & 1.44 & 1.77 & 1.41 & 1.13 & 1.29 & 1.48 \\
\hline Th & 2.33 & 1.78 & 2.09 & 2.20 & 2.06 & 2.08 & 2.10 & 1.77 & 2.05 & 1.84 & 2.54 & 2.39 & 1.70 & 2.17 & 2.18 \\
\hline U & 0.55 & 0.51 & 0.46 & 0.50 & 0.50 & 0.46 & 0.52 & 0.47 & 0.51 & 0.50 & 0.63 & 0.51 & 0.47 & 0.58 & 0.55 \\
\hline
\end{tabular}




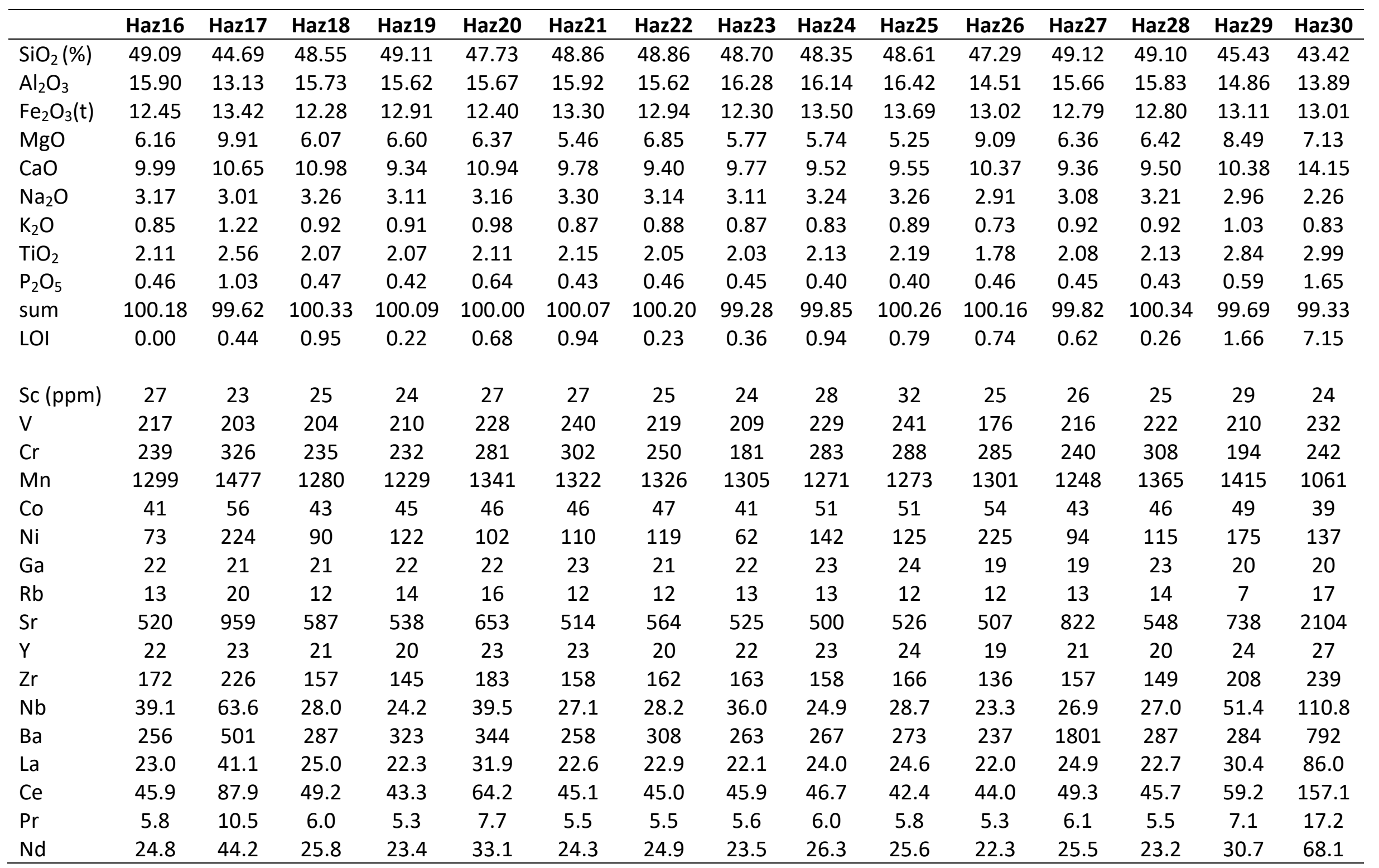




\begin{tabular}{|c|c|c|c|c|c|c|c|c|c|c|c|c|c|c|c|}
\hline & Haz16 & Haz17 & Haz18 & Haz19 & Haz20 & Haz21 & Haz22 & Haz23 & Haz24 & Haz25 & Haz26 & Haz27 & Haz28 & Haz29 & Haz30 \\
\hline $\mathrm{Sm}$ & 5.33 & 8.92 & 5.87 & 5.30 & 6.43 & 5.29 & 5.26 & 5.01 & 5.33 & 5.93 & 5.30 & 5.14 & 4.89 & 6.10 & 12.45 \\
\hline $\mathrm{Eu}$ & 1.94 & 3.00 & 1.95 & 1.95 & 2.22 & 1.91 & 1.79 & 1.83 & 1.86 & 2.05 & 1.69 & 1.76 & 1.76 & 2.16 & 3.80 \\
\hline $\mathrm{Gd}$ & 6.23 & 7.49 & 5.08 & 4.96 & 6.03 & 5.36 & 4.60 & 4.95 & 5.78 & 6.04 & 5.12 & 5.29 & 4.74 & 6.29 & 9.84 \\
\hline $\mathrm{Tb}$ & 0.84 & 0.92 & 0.68 & 0.63 & 0.85 & 0.76 & 0.69 & 0.75 & 0.72 & 0.84 & 0.68 & 0.73 & 0.68 & 0.85 & 1.16 \\
\hline Dy & 4.59 & 5.07 & 4.19 & 4.14 & 4.91 & 4.64 & 3.91 & 4.27 & 4.86 & 4.55 & 4.25 & 4.37 & 4.16 & 4.79 & 6.31 \\
\hline Ho & 0.88 & 0.84 & 0.79 & 0.68 & 0.88 & 0.85 & 0.73 & 0.77 & 1.00 & 0.94 & 0.75 & 0.75 & 0.75 & 0.93 & 1.00 \\
\hline $\mathrm{Er}$ & 2.11 & 2.15 & 2.06 & 2.01 & 1.99 & 2.33 & 1.99 & 2.09 & 2.25 & 2.49 & 1.76 & 2.00 & 1.97 & 2.46 & 2.37 \\
\hline $\mathrm{Tm}$ & 0.28 & 0.26 & 0.23 & 0.29 & 0.29 & 0.27 & 0.27 & 0.25 & 0.32 & 0.34 & 0.22 & 0.26 & 0.23 & 0.30 & 0.30 \\
\hline $\mathrm{Yb}$ & 1.71 & 1.61 & 1.70 & 1.51 & 1.72 & 1.86 & 1.73 & 1.76 & 1.83 & 1.86 & 1.60 & 1.64 & 1.70 & 1.76 & 1.79 \\
\hline Lu & 0.21 & 0.19 & 0.21 & 0.22 & 0.22 & 0.22 & 0.22 & 0.26 & 0.22 & 0.23 & 0.16 & 0.21 & 0.21 & 0.26 & 0.24 \\
\hline $\mathrm{Hf}$ & 3.87 & 4.76 & 3.67 & 3.22 & 3.77 & 3.57 & 3.89 & 3.64 & 3.53 & 4.01 & 2.86 & 3.55 & 3.41 & 4.66 & 5.11 \\
\hline $\mathrm{Ta}$ & 2.04 & 3.08 & 1.38 & 1.27 & 2.22 & 1.41 & 1.62 & 1.75 & 1.27 & 1.41 & 1.42 & 1.30 & 1.30 & 2.83 & 4.96 \\
\hline Th & 1.93 & 3.09 & 2.17 & 1.94 & 2.91 & 2.05 & 2.35 & 1.95 & 2.01 & 2.14 & 1.98 & 2.23 & 1.94 & 3.39 & 7.57 \\
\hline$U$ & 0.51 & 0.83 & 0.45 & 0.40 & 0.89 & 0.52 & 0.58 & 0.69 & 0.53 & 0.58 & 0.53 & 0.52 & 0.53 & 1.05 & 1.91 \\
\hline
\end{tabular}




\begin{tabular}{|c|c|c|c|c|c|c|c|c|c|c|c|c|c|c|c|}
\hline & Haz31 & Haz32 & Haz33 & Haz34 & Haz35 & Haz36 & Haz37 & Haz38 & Haz39 & Haz40 & Haz41 & Haz42 & Haz43 & Haz44 & RMS \\
\hline $\mathrm{SiO}_{2}(\%)$ & 46.91 & 47.63 & 48.24 & 48.80 & 48.77 & 49.11 & 48.14 & 48.67 & 48.73 & 49.10 & 49.09 & 47.80 & 49.23 & 49.02 & 0.4 \\
\hline $\mathrm{Al}_{2} \mathrm{O}_{3}$ & 14.22 & 14.91 & 15.96 & 15.74 & 15.57 & 16.35 & 15.52 & 15.66 & 16.10 & 16.58 & 16.12 & 14.89 & 16.67 & 15.79 & 0.5 \\
\hline $\mathrm{Fe}_{2} \mathrm{O}_{3}(\mathrm{t})$ & 12.54 & 12.61 & 12.35 & 12.42 & 13.25 & 12.92 & 14.45 & 12.85 & 13.16 & 12.92 & 12.74 & 13.04 & 12.17 & 12.94 & 0.6 \\
\hline $\mathrm{MgO}$ & 9.36 & 7.86 & 5.03 & 6.53 & 6.80 & 5.88 & 6.77 & 6.62 & 5.82 & 4.93 & 6.37 & 8.31 & 5.47 & 6.60 & 0.4 \\
\hline $\mathrm{CaO}$ & 9.75 & 10.18 & 12.21 & 9.82 & 9.43 & 9.30 & 9.15 & 9.97 & 9.30 & 9.53 & 9.74 & 9.94 & 10.07 & 9.34 & 0.4 \\
\hline $\mathrm{Na}_{2} \mathrm{O}$ & 2.42 & 2.96 & 3.07 & 3.25 & 3.05 & 3.25 & 3.03 & 3.11 & 3.17 & 3.41 & 3.22 & 2.71 & 3.23 & 3.14 & 1.0 \\
\hline $\mathrm{K}_{2} \mathrm{O}$ & 1.25 & 0.95 & 0.86 & 0.91 & 0.80 & 0.89 & 0.85 & 0.86 & 0.91 & 1.04 & 0.81 & 0.83 & 0.92 & 0.92 & 0.5 \\
\hline $\mathrm{TiO}_{2}$ & 2.53 & 2.13 & 2.29 & 2.13 & 2.08 & 2.10 & 2.01 & 2.06 & 2.10 & 2.24 & 2.05 & 1.90 & 2.04 & 2.13 & 0.5 \\
\hline $\mathrm{P}_{2} \mathrm{O}_{5}$ & 0.65 & 0.36 & 0.48 & 0.48 & 0.33 & 0.35 & 0.42 & 0.41 & 0.34 & 0.54 & 0.29 & 0.44 & 0.45 & 0.40 & 1.1 \\
\hline sum & 99.63 & 99.59 & 100.49 & 100.08 & 100.08 & 100.15 & 100.34 & 100.21 & 99.63 & 100.29 & 100.43 & 99.86 & 100.25 & 100.28 & \\
\hline LOI & 2.22 & 0.56 & 2.42 & -0.17 & 0.62 & 0.87 & 0.75 & 0.72 & 1.14 & 0.89 & -0.01 & 0.65 & 0.32 & 0.66 & \\
\hline Sc (ppm) & 24 & 27 & 27 & 25 & 26 & 27 & 26 & 22 & 23 & 27 & 25 & 27 & 24 & 26 & \\
\hline V & 202 & 251 & 380 & 214 & 256 & 252 & 265 & 245 & 219 & 287 & 308 & 231 & 256 & 237 & \\
\hline $\mathrm{Cr}$ & 247 & 408 & 145 & 246 & 313 & 230 & 353 & 219 & 200 & 255 & 205 & 336 & 170 & 269 & \\
\hline $\mathrm{Mn}$ & 1246 & 1545 & 1543 & 1324 & 1503 & 1603 & 1589 & 1630 & 1669 & 1490 & 1481 & 1476 & 1271 & 1498 & \\
\hline Co & 49 & 60 & 46 & 43 & 52 & 53 & 66 & 53 & 57 & 49 & 48 & 55 & 40 & 51 & \\
\hline $\mathrm{Ni}$ & 190 & 236 & 55 & 96 & 149 & 128 & 203 & 141 & 136 & 107 & 85 & 223 & 60 & 125 & \\
\hline $\mathrm{Ga}$ & 20 & 23 & 28 & 27 & 24 & 26 & 26 & 28 & 26 & 27 & 26 & 22 & 26 & 24 & \\
\hline $\mathrm{Rb}$ & 14 & 18 & 14 & 21 & 11 & 15 & 17 & 19 & 17 & 15 & 13 & 12 & 17 & 13 & \\
\hline $\mathrm{Sr}$ & 623 & 504 & 588 & 729 & 517 & 610 & 558 & 584 & 575 & 589 & 481 & 534 & 591 & 599 & \\
\hline$Y$ & 22 & 33 & 24 & 20 & 29 & 19 & 21 & 19 & 18 & 28 & 20 & 29 & 23 & 28 & \\
\hline $\mathrm{Zr}$ & 177 & 149 & 197 & 147 & 138 & 161 & 160 & 153 & 132 & 185 & 122 & 135 & 182 & 160 & \\
\hline $\mathrm{Nb}$ & 37.3 & 34.1 & 46.6 & 25.3 & 23.9 & 27.5 & 31.0 & 26.5 & 22.3 & 34.5 & 24.5 & 26.4 & 35.7 & 28.1 & \\
\hline $\mathrm{Ba}$ & 218 & 406 & 277 & 421 & 308 & 340 & 343 & 343 & 424 & 352 & 264 & 247 & 301 & 340 & \\
\hline La & 27.7 & 16.6 & 23.8 & 24.3 & 17.6 & 20.2 & 21.9 & 21.1 & 20.0 & 29.7 & 16.4 & 21.9 & 22.1 & 21.4 & \\
\hline $\mathrm{Ce}$ & 56.1 & 40.4 & 58.3 & 54.5 & 38.8 & 44.7 & 46.7 & 51.0 & 50.4 & 59.1 & 35.6 & 49.3 & 46.2 & 48.6 & \\
\hline $\operatorname{Pr}$ & 6.8 & 4.5 & 6.8 & 5.9 & 4.6 & 5.0 & 5.5 & 5.5 & 5.2 & 7.2 & 4.1 & 5.6 & 5.7 & 5.5 & \\
\hline $\mathrm{Nd}$ & 29.3 & 19.9 & 30.6 & 25.6 & 20.6 & 22.0 & 23.2 & 23.4 & 22.6 & 31.3 & 19.5 & 24.6 & 24.6 & 24.4 & \\
\hline
\end{tabular}




\begin{tabular}{|c|c|c|c|c|c|c|c|c|c|c|c|c|c|c|c|}
\hline & Haz31 & Haz32 & Haz33 & Haz34 & Haz35 & Haz36 & Haz37 & Haz38 & Haz39 & Haz40 & Haz41 & Haz42 & Haz43 & Haz44 & RMS \\
\hline $\mathrm{Sm}$ & 6.55 & 4.68 & 6.33 & 5.79 & 4.83 & 4.51 & 4.93 & 4.75 & 4.87 & 6.58 & 4.35 & 5.40 & 5.67 & 5.11 & \\
\hline $\mathrm{Eu}$ & 2.31 & 1.62 & 2.24 & 1.95 & 1.68 & 1.84 & 1.83 & 1.83 & 1.85 & 2.17 & 1.70 & 1.75 & 1.89 & 1.80 & \\
\hline $\mathrm{Gd}$ & 5.95 & 4.40 & 6.52 & 5.46 & 4.47 & 4.53 & 5.03 & 4.84 & 4.42 & 6.90 & 4.43 & 4.90 & 5.44 & 4.94 & \\
\hline $\mathrm{Tb}$ & 0.83 & 0.54 & 0.93 & 0.74 & 0.63 & 0.62 & 0.69 & 0.58 & 0.60 & 0.89 & 0.53 & 0.74 & 0.75 & 0.65 & \\
\hline Dy & 4.39 & 3.58 & 5.24 & 4.87 & 3.86 & 3.66 & 4.44 & 3.87 & 3.95 & 5.48 & 3.18 & 4.14 & 4.49 & 3.96 & \\
\hline Ho & 0.77 & 0.57 & 0.87 & 0.66 & 0.74 & 0.69 & 0.78 & 0.70 & 0.60 & 1.02 & 0.57 & 0.70 & 0.85 & 0.71 & \\
\hline $\mathrm{Er}$ & 1.99 & 1.69 & 2.43 & 2.08 & 2.18 & 1.78 & 2.17 & 2.07 & 1.96 & 2.72 & 1.44 & 2.03 & 2.32 & 1.91 & \\
\hline $\mathrm{Tm}$ & 0.22 & 0.22 & 0.29 & 0.23 & 0.24 & 0.24 & 0.25 & 0.25 & 0.20 & 0.32 & 0.19 & 0.22 & 0.30 & 0.25 & \\
\hline $\mathrm{Yb}$ & 1.50 & 1.36 & 1.98 & 1.54 & 1.63 & 1.66 & 1.72 & 1.28 & 1.42 & 2.01 & 1.44 & 1.68 & 1.91 & 1.66 & \\
\hline Lu & 0.19 & 0.16 & 0.24 & 0.21 & 0.21 & 0.18 & 0.23 & 0.22 & 0.22 & 0.26 & 0.20 & 0.24 & 0.24 & 0.25 & \\
\hline $\mathrm{Hf}$ & 3.97 & 3.46 & 4.77 & 3.21 & 3.29 & 3.66 & 3.67 & 3.37 & 2.97 & 4.50 & 2.94 & 3.22 & 4.05 & 3.75 & \\
\hline Тa & 1.89 & 1.68 & 2.16 & 1.17 & 1.14 & 1.60 & 1.51 & 1.19 & 1.04 & 1.67 & 1.09 & 1.30 & 1.62 & 1.36 & \\
\hline Th & 2.57 & 1.68 & 2.37 & 2.28 & 1.68 & 2.00 & 1.94 & 2.00 & 1.81 & 2.53 & 2.21 & 2.06 & 1.98 & 2.04 & \\
\hline$U$ & 0.73 & 0.45 & 0.63 & 0.70 & 0.54 & 0.64 & 0.62 & 0.70 & 0.51 & 0.75 & 0.50 & 0.43 & 0.70 & 0.59 & \\
\hline
\end{tabular}




\section{Results}

\subsection{Major and trace element composition}

The main goal of the present study is to use the geochemical data to determine whether or not multiple vessels were produced from a single basaltic rock source that represents a particular extraction site. However, products from a single extraction site will never show an identical geochemical composition because they always mirror the natural heterogeneity within a rock outcrop (Gluhak \& Hofmeister 2009; 2011; Gluhak \& Rosenberg 2013). Thus, two or more rocks can only be defined as the same on the basis of the similarity of their geochemical compositions, with "similarity" meaning a low variation in geochemical features.

According to the IUGS-recommendations for classification of fine-grained volcanic rocks (Le Maitre et al. 1989), the silica and alkali content of the samples are plotted in the TAS-diagram (Le Bas et al. 1986) (see Figure 2).

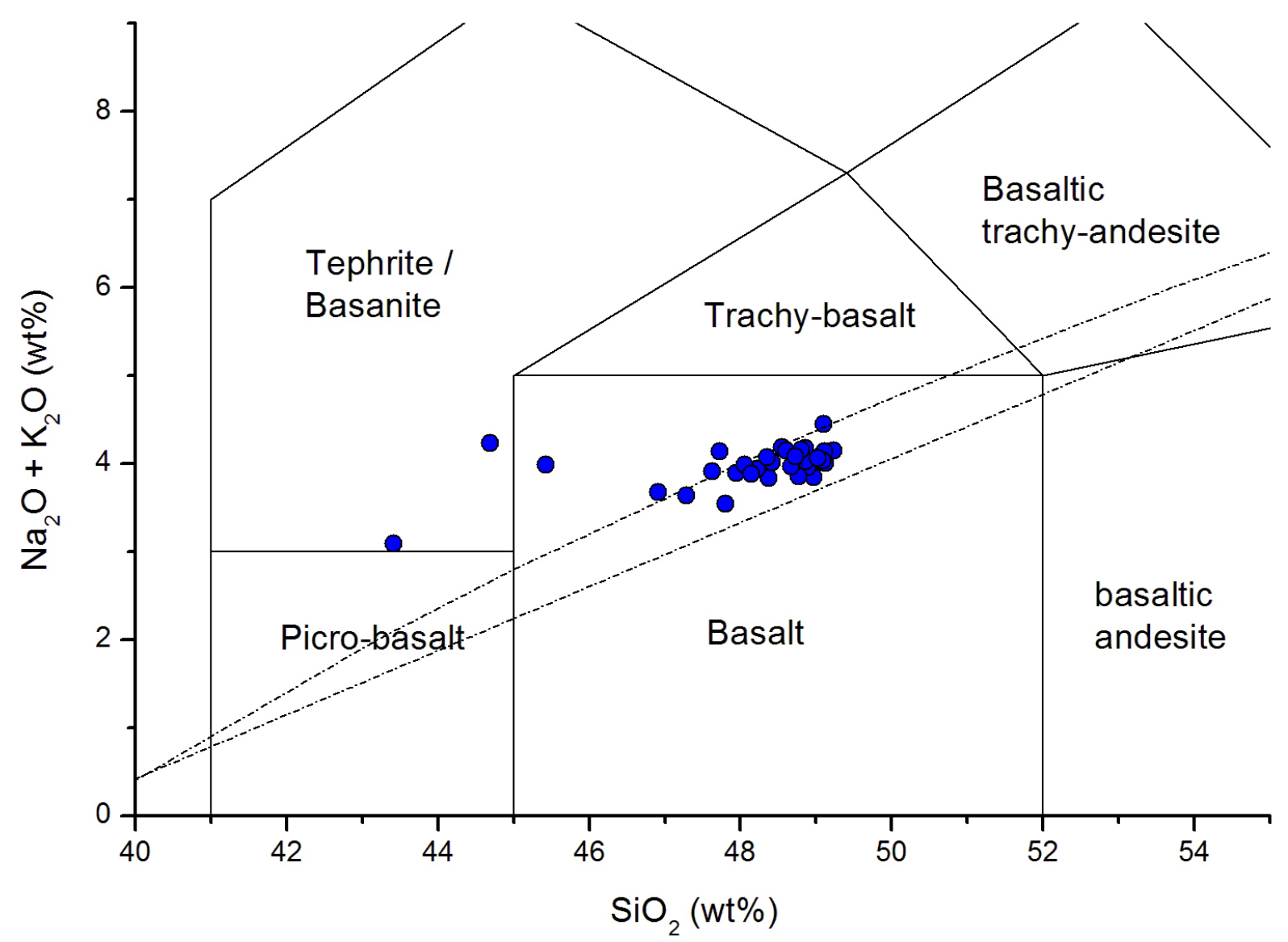

Figure 2. Classification of the samples in the TAS-diagram.

The bulk of the samples are slightly alkaline basalts sensu stricto. Two samples are basanites according to their normative (i.e. CIPW-norm calculated) olivine content of greater than $10 \%$. Thirty-three of the vessels contain normative hypersthene up to $8.25 \%$, and the other eleven contain normative nepheline, up to $6.79 \%$ (Table 3). The fact that the majority of vessels plot as a cluster in the basalt field of the TAS-diagram already hints at the use of a specific, preferred basalt occurrence. However, the difficulty in determining whether or not the majority of vessels were produced from the same outcrop, meaning from one (or more than one) extraction site, is shown in Figures $3 \mathrm{a}$ and $3 \mathrm{~b}$ (Mg\# versus $\mathrm{Al}_{2} \mathrm{O}_{3}$ and $\mathrm{Mg \#}$ versus $\mathrm{CaO}$ ). These figures show that the "basalt group," which seems well-constrained in the TAS- 


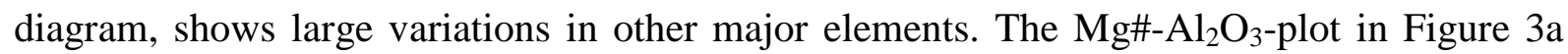
e.g., displays the natural trend of a typical magmatic differentiation, from high Mg\# - low alumina-content to low Mg\# - high alumina-content, a trend that can display a general trend in basaltic rocks from a certain region but can also be present within a single basalt occurrence. Therefore, it cannot be determined based on the major element composition of the vessels alone if these variations are due to the natural inhomogeneity within one extraction site or if they indicate that the basalt vessels originate from more than one extraction site.

Table 3. CIPW-normative mineral content.

\begin{tabular}{|c|c|c|c|c|}
\hline & Av. & Min. & Max. & No. \\
\hline Or & 5.31 & 4.31 & 7.39 & \\
\hline$A b$ & 24.76 & 10.87 & 28.86 & \\
\hline An & 25.92 & 18.71 & 28.27 & \\
\hline Di & 17.02 & 13.65 & 28.12 & \\
\hline Hy & 3.19 & 1.12 & 8.25 & 33 \\
\hline Ol & 16.26 & 11.74 & 22.56 & \\
\hline $\mathrm{mt}$ & 1.87 & 1.77 & 2.10 & \\
\hline II & 4.05 & 3.38 & 5.68 & \\
\hline Ap & 1.05 & 0.63 & 3.60 & \\
\hline ne & 0.71 & 1.32 & 6.79 & 11 \\
\hline
\end{tabular}

Trends of certain trace elements show us that the lavas were probably subjected to varying degrees of fractional crystallisation (Figure 4) during their development. The trace element patterns of the samples are presented in Figure 5. As intraplate volcanic rocks, characteristic for this region, the samples display the typical pattern of ocean island basalts (OIB). Again, the majority of samples display a very similar geochemical pattern. Two samples show higher trace element concentrations, a typical phenomenon for basanite rocks of the region. Thus, the trace elements offer even fewer characteristics to constrain rocks from each other than the major elements.

\subsection{Multivariate evaluation}

To determine if a Tel Hazor "core group” representative of a certain extraction site is present in our set of samples, we needed to decide which geochemical features within our group of measured elements are characteristic, i.e. which geochemical features can be used to constrain potential groups from each other. To do this, a principal component analysis (PCA) was conducted; although in the present study we do not have the ideal number of cases available for PCA (the number of cases should exceed at least three times the number of variables). All measured elements were entered as variables. Prior to PCA the data were logtransformed to ensure the independency of the compositional data and z-standardized, resulting in a mean of 0 and a standard deviation of 1 . The PCA was calculated with VARIMAX and PROMAX rotation, which had no influence on the following results: seven principal components (PCs) were extracted, which explains about $87 \%$ of the total variance, with the first three PCs explaining about $66 \%$. The elements with communalities of more than 0.9 after the PCs' extraction are $\mathrm{SiO}_{2}, \mathrm{Al}_{2} \mathrm{O}_{3}, \mathrm{P}_{2} \mathrm{O}_{5}, \mathrm{Co}, \mathrm{Ni}, \mathrm{Sr}, \mathrm{Zr}, \mathrm{Nb}, \mathrm{La}, \mathrm{Ce}, \mathrm{Pr}, \mathrm{Nd}, \mathrm{Sm}, \mathrm{Eu}$, Gd, Tb, Ho, and Hf, with $\mathrm{P}_{2} \mathrm{O}_{5}$, La and Pr showing the highest communalities, which means that the extracted PCs represent these variables well. 

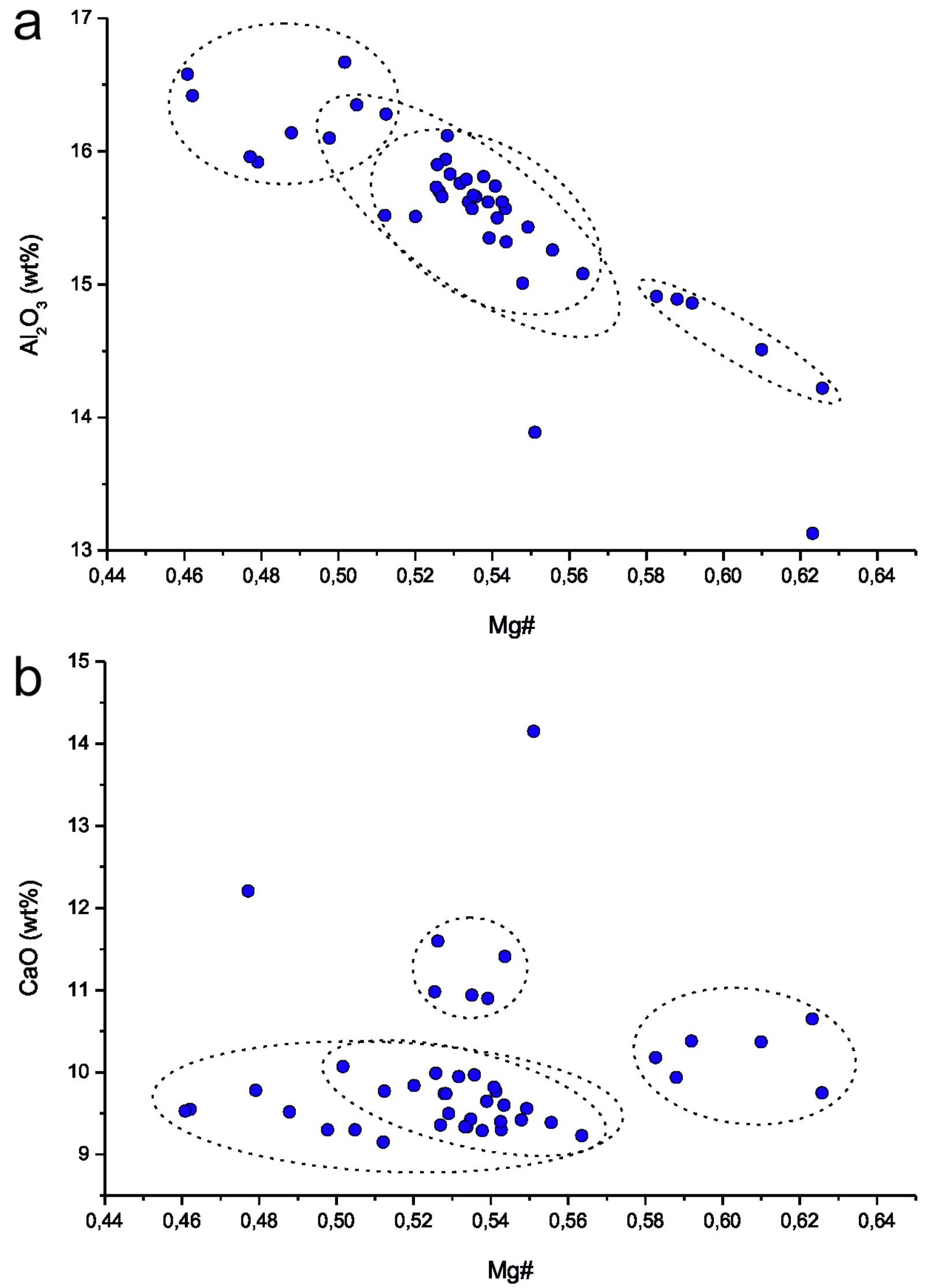

Figure 3. a) Magnesium-number $\mathrm{Mg \#}$ versus $\mathrm{Al} 2 \mathrm{O} 3$ and $\mathrm{Mg \#}$ versus $\mathrm{CaO}$, with $\mathrm{Mg \#} \mathrm{=}$ $(\mathrm{MgO} / 40.32) /[(\mathrm{MgO} / 40.32+\mathrm{Fe} 2 \mathrm{O} 3(\mathrm{t}) \cdot 0.9 / 79.8)]$ (Harvey et al. 2006). The stippled lines indicate possible groupings. 


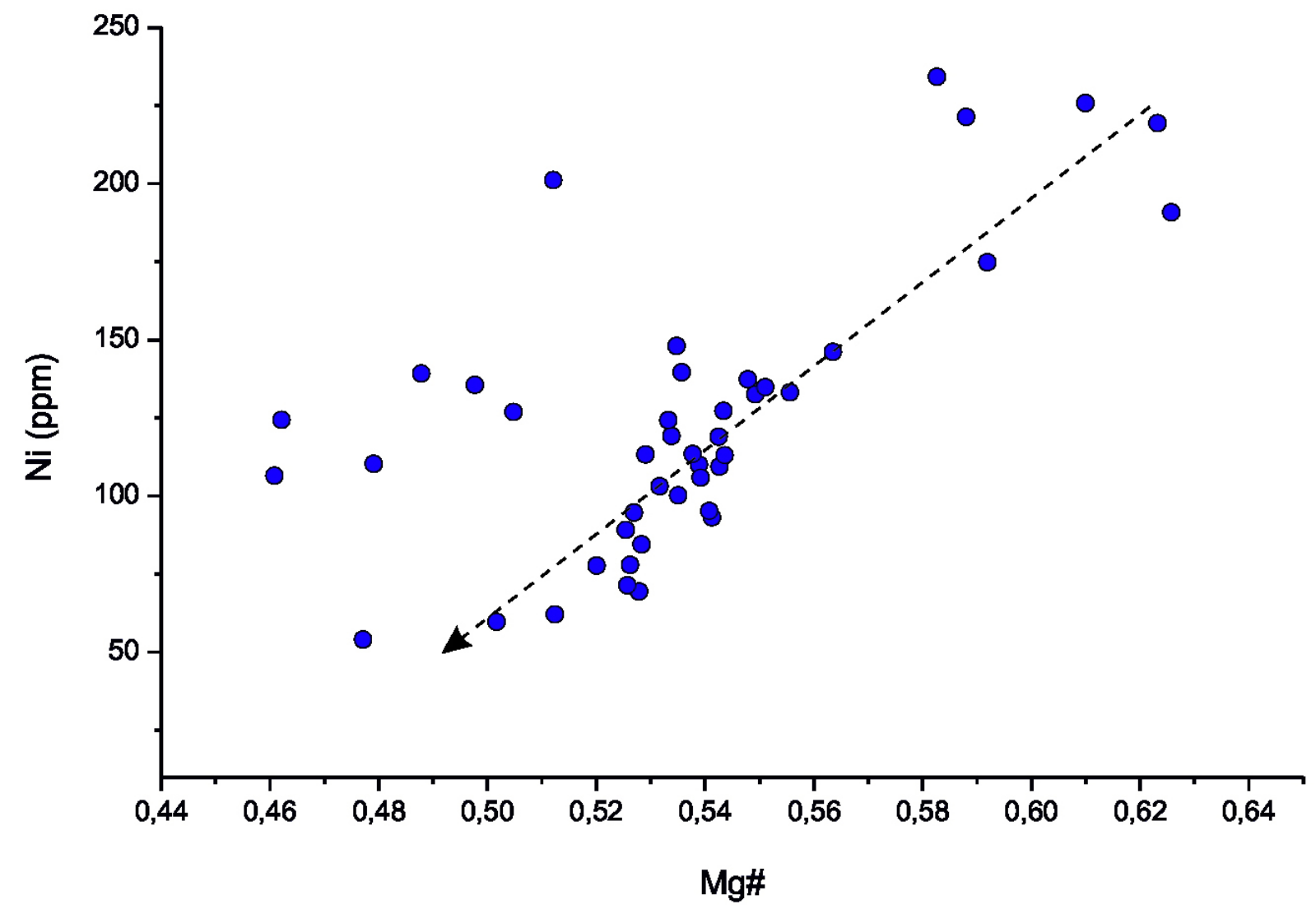

Figure 4. Magnesium-number (Mg\#) versus Ni. Mg\# see Figure 3

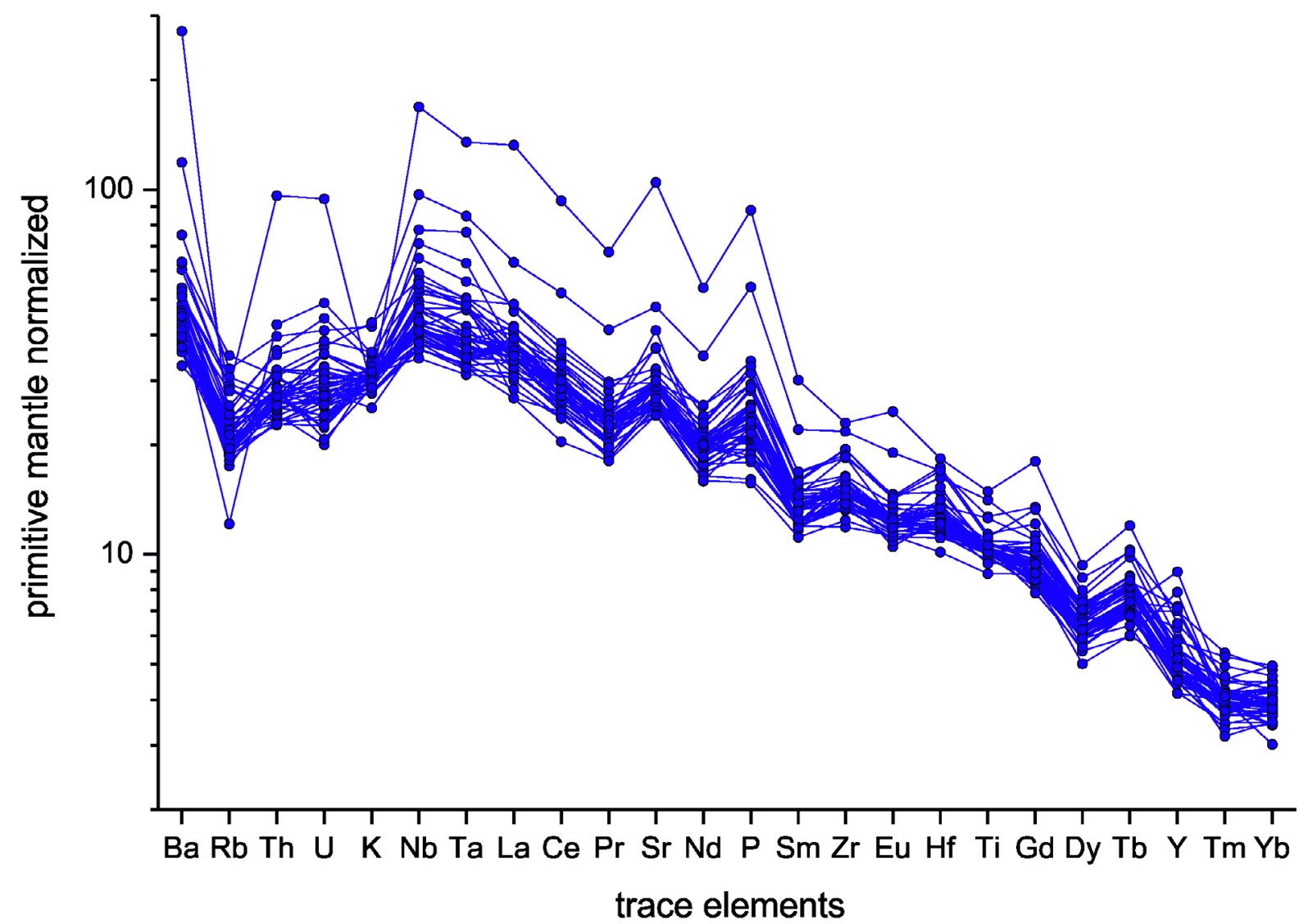

Figure 5. Primitive mantle-normalized trace element diagram. Primitive mantle-values taken from McDonough and Sun (1995). 
In the following step the log-transformed data of these elements were used as variables for cluster analyses (CA) of the basalt vessels. The CAs were calculated with average linkage as cluster algorithm, combined with squared Euclidian Distance and city block distance as distance measures, as well as with the Ward-algorithm with squared Euclidean distance (Figure 6). All cluster procedures indicate that the same 36 of the 44 samples are always affiliated into one group. Seven samples do not belong to this group. However, five of these "outsiders" are affiliated into one cluster by all cluster methods. Two samples, both basanites from Figure 1, show no similarity with any of the other samples. The cluster results only disagree in sample Haz43: where the average linkage algorithm places it into the group of the large majority, the Ward algorithm places it in the "outsider" group.

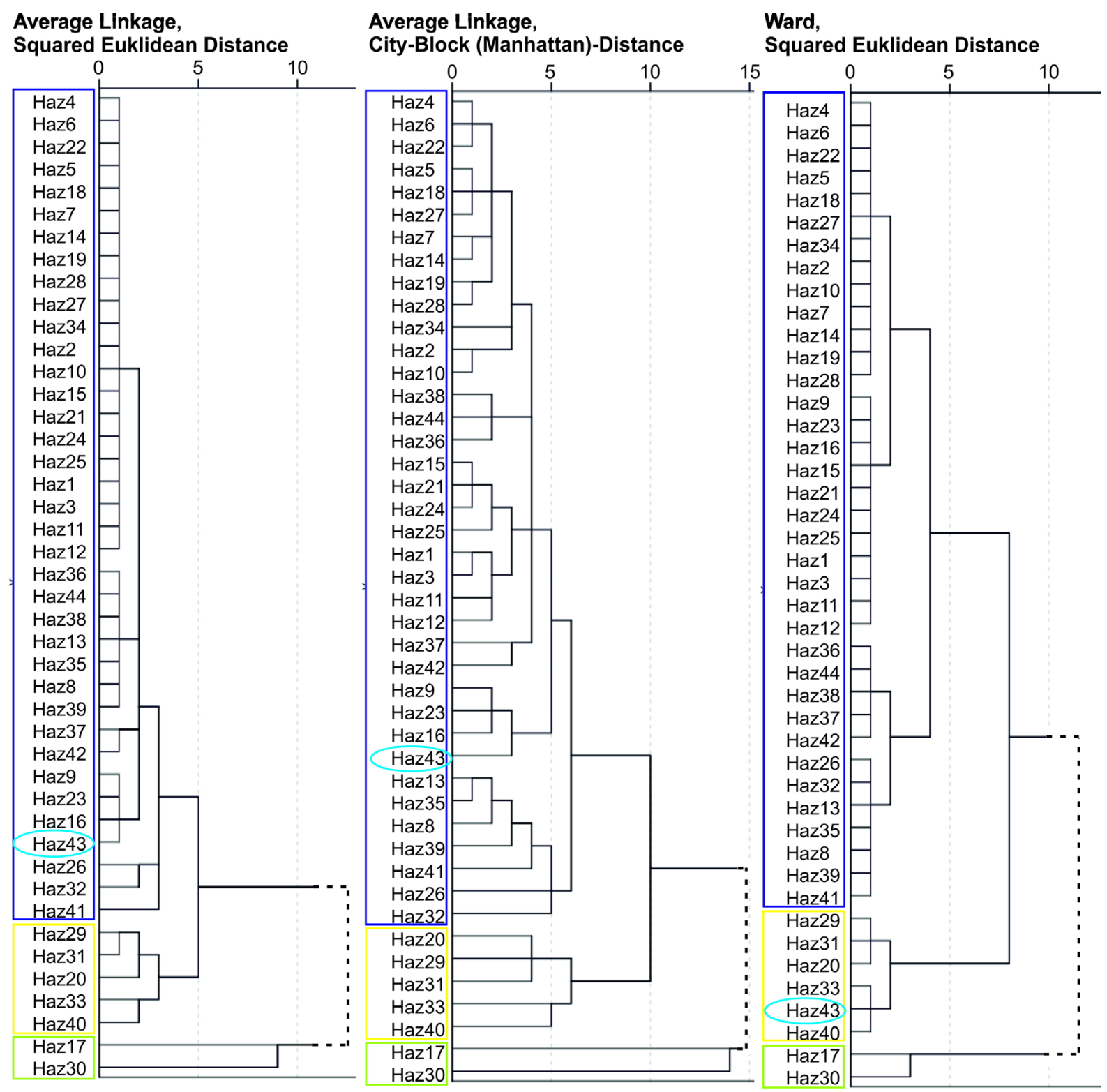

Figure 6. Cluster analysis results. The Tel Hazor reference group is marked with a blue frame and the vessels representing a potential second extraction site are marked with a yellow frame. The two individuals are marked in green. Haz43 is marked with a light blue ellipse.

Figure 7 presents a combination of the information gained from PCA and CA. The spots are coloured according to their cluster affiliation on a level of three clusters. Based on the first two principal components ("factors", Figure 7), the dominant group is displayed as a well- 
constrained cluster. PCA determined $\mathrm{P}_{2} \mathrm{O}_{5}$, La and $\mathrm{Pr}$ as elements with highest communalities. A plot of the log transformed concentrations of these elements (Figure 8) shows in the threedimensional case a confined dominant geochemical group within the vessels. In both Figures 7 and 8, sample Haz43 plots within this dominant group.

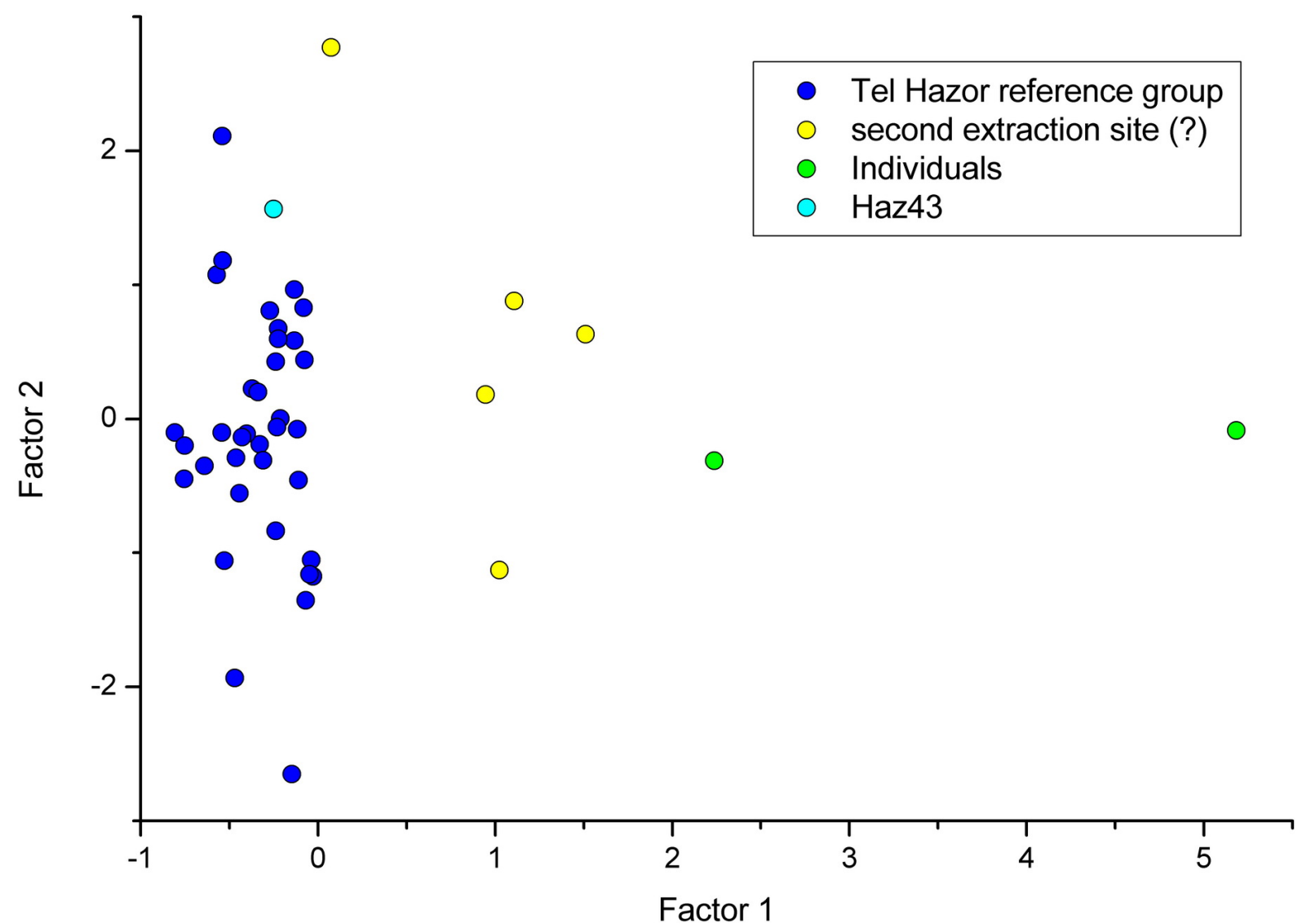

Figure 7. Combination of PCA and CA results: First two principal components. The dark blue spots are the samples clustered into the dominant Tel Hazor group. The light blue spot is sample Haz43. The yellow samples possibly represent a second minor extraction site. The green samples are the basanites, which show no connection to any others.

\section{Discussion and conclusion}

Previous geochemical-mineralogical studies of basalt artefacts in the southern Levant concentrated mainly on the determination of the provenance of the artefacts, but these studies always had rather limited results due to the fact that basaltic rock extraction sites had not been identified (apart from the Late Neolithic-Early Chalcolithic extraction site at Giv'at Kipod, see Rosenberg et al. 2008; Gluhak \& Rosenberg 2013; Rosenberg \& Gluhak 2016; Shimelmitz \& Rosenberg 2016) and that geochemical data from geological samples for comparison were, if available at all, only superficially included (e.g., Amiran \& Porat 1984; Philip \& Williams-Thorpe 1993; 2000; 2001; Williams-Thorpe \& Thorpe 1993; Rowan 1998; Rutter 2003; Rutter et al. 2003; Watts et al. 2004; Rutter \& Philip 2008).

The potential for determining the raw material variability within an assemblage of basaltic rock artefacts and its use as a baseline for determine artefact provenance seems so far underestimated. It provides important information to investigate distribution networks, even when the actual, final extraction site in the field remains (as yet) unknown. The basalt vessel workshop at Tel Hazor offers the possibility of studying a large number of samples to reconstruct the site's role in the production and distribution of basalt vessels. The archaeological question concerning raw material variability within an assemblage of rock 
artefacts can be "translated" into the question of how to find a way to determine which rocks are similar to each other and which are the decisive parameters. Since a thin section study of the raw material was only possible in a few cases, we concentrate on a thorough evaluation of major and trace element data combined with multivariate methods in the present study.

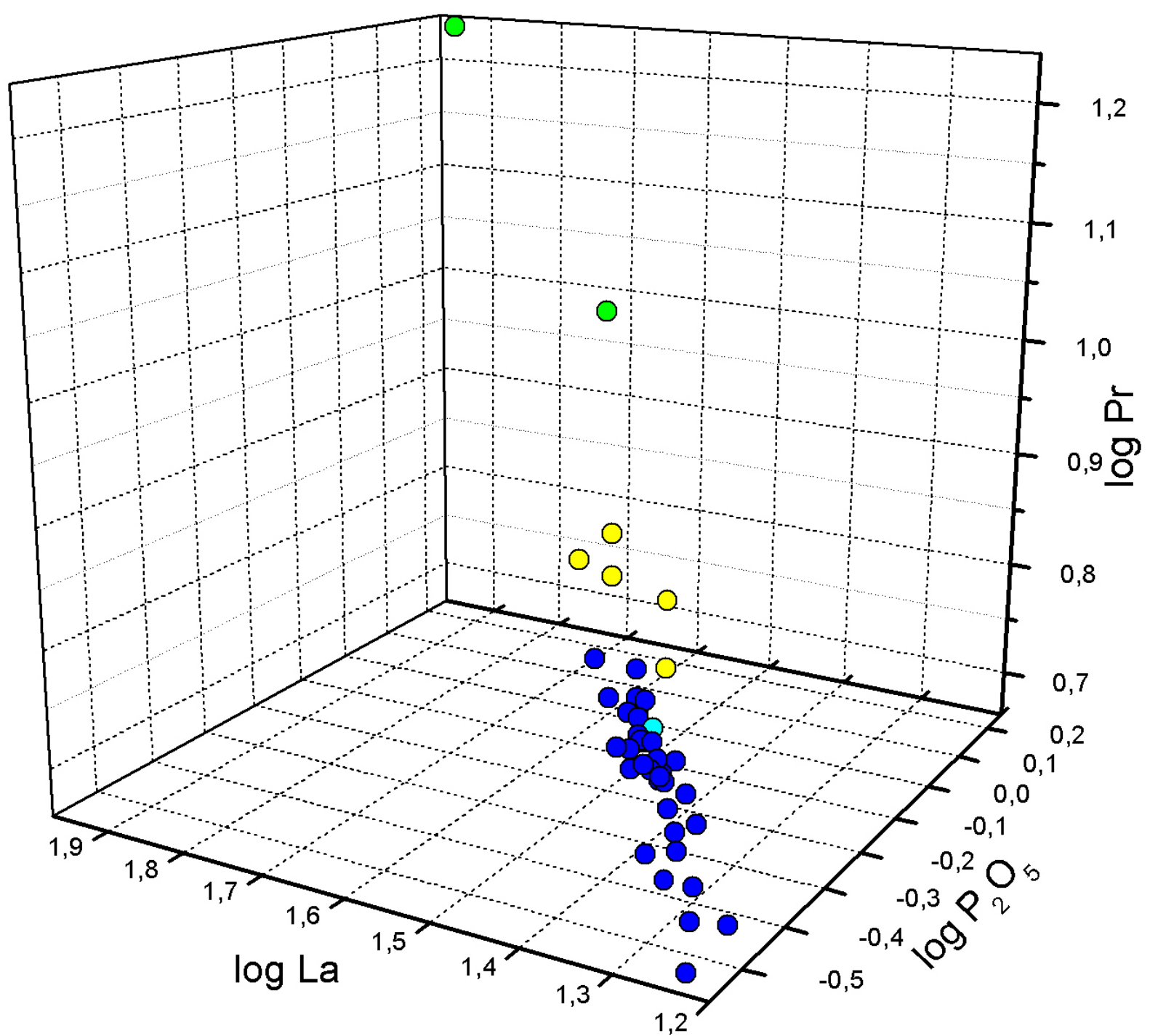

Figure 8. Combination of PCA and CA results: The log-transformed values of La, P2O5 and Pr. Spot colours as in Figure 7.

The data show that the Tel Hazor workshop was mainly provided with raw material from one specific extraction site, implying a specific selection of raw material. A second, minor extraction site might have also existed. Only two of 44 samples show no connection to all other samples and come from other places. Our results thus suggest that, during the Late Bronze Age and the Iron Age, craftspeople at Hazor preferred a specific, as yet unknown, basalt source, located not in the immediate surroundings, but near a site on the Korazim block. However, this assumption has yet to be tested, and, to that end, a study of geological samples from the field is currently being undertaken. In turn, we can use the results to establish a connection between the products of the Iron Age workshop and basalt vessels found in other contexts. These results point to long-term raw material preference and access that bridged the Late Bronze and Iron Age occupation of the tell and may suggest a connection between the Bronze and Iron Age craftspeople at Hazor. 
The preference for a specific basalt source is striking given the range of sources in the vicinity of Tel Hazor. These preferences, however, were probably governed by several factors. These might include the raw material properties ('quality'), transportation costs, quarry rights over specific outcrops, and others. While field data are still being analyzed to establish the location of the specific extraction site, it is already possible to use the "Tel Hazor reference group" (the characteristics of the 36 samples that represent this major extraction site) as a basis to investigate if Iron Age (as well as Late Bronze Age) basalt vessels found at other sites in the Levant were indeed produced at Hazor or if there is evidence for other workshops during this time period. These future results can help us assess what role Tel Hazor played in the production and distribution of basalt vessels during the Late Bronze and Iron Age and determine if the site was an important centre for basalt vessel production and trade or if the craftspeople working in the Area M workshop manufactured products for Hazor only.

\section{Acknowledgements}

The authors thank the current director of the Selz Foundation Hazor Excavations in Memory of Yigael Yadin, A. Ben-Tor, and co-director of the Hazor Excavations, the late S. Zuckerman, for permission to study the basalt vessels from Hazor. We would also like to thank supervisor of Area M, S. Bechar, for her assistance with this project. Financial support was provided by an Alumni Research and Scholarly Activity Fellowship from the University of Evansville.

\section{References}

Amiran, R., \& Porat, N. 1984, The basalt vessels of the Chalcolithic period and Early Bronze Age I. Tel Aviv, 11(1): 11-19. doi:10.1179/tav.1984.1984.1.11

Ben-Tor, A., (Ed.), 1989, Hazor III-IV, Text. Israel Exploration Society, Jerusalem, 362 p.

Ben-Tor, A., \& Bonfil, R., (Eds.), 1997, Hazor V: An Account of the Fifth Season of Excavation, 1968. Israel Exploration Society, Jerusalem, 408 p.

Ben-Tor, A., \& Zuckerman, S. 2010, Tel Hazor-2010 preliminary report. Hadashot Arkheologiyot-Excavations and Surveys in Israel, 122. URL: http://www.hadashotesi.org.il/report_detail_eng.aspx?id=1592

Bevan, A. 2007. Stone Vessels and Values in the Bronze Age Mediterranean. University College London, Institute of Archaeology. Cambridge, 316 p. doi:10.1017/CBO9780511499678

Ebeling, J. 2012, Ground stone artifacts. In: Hazor VI: The 1990-2009 Excavations, the Iron Age (Ben-Tor, A., Ben-Ami, D., \& Sandhaus, D., Eds.), Israel Exploration Society, Jerusalem: p. 542-558.

Ebeling, J., \& Rosenberg, D. 2015. A basalt vessel workshop and its products at Iron Age Hazor. Journal of Field Archaeology, 40(6): 665-674. doi:10.1080/00934690.2015.1101941

Gluhak, T.M., \& Hofmeister, W. 2009, Roman lava quarries in the Eifel region (Germany): geochemical data for millstone provenance studies. Journal of Archaeological Science, 36(8): 1174-1182. doi:10.1016/j.jas.2009.04.007 
Gluhak, T.M., \& Hofmeister, W. 2011, Geochemical provenance analyses of Roman lava millstones north of the Alps: a study of their distribution and implications for the beginning of Roman lava quarrying in the Eifel region (Germany). Journal of Archaeological Science, 38(7): 1603-1620. doi:10.1016/j.jas.2011.02.025

Gluhak, T.M., \& Rosenberg, D. 2013, Geochemical discrimination of basaltic sources as a tool for provenance analyses of bifacial tools in the southern Levant: first results from the Jezreel Valley, Israel. Journal of Archaeological Science, 40: 1611-1622. doi:10.1016/j.jas.2012.11.003

Harvey, J., Gannoun, A., Burtin, K.W., Rogers, N.W., Alard, O., \& Parkinson, I.J. 2006, Ancient melt extraction from the oceanic upper mantle revealed by Re-Os isotopes in abyssal peridotites from the Mid-Atlantic ridge. Earth and Planetary Science Letters, 244(3-4): 606-621. doi:10.1016/j.epsl.2006.02.031

Jochum, K.P., Nohl, U., Herwig, K., Lammel, E., Stoll, B., \& Hofmann, A.W. 2005, GeoReM: a new geochemical database for reference materials and isotopic standards. Geostandards and Geoanalytical Research, 29(3): 333-338. doi:10.1111/j.1751908x.2005.tb00904.x

Le Bas, M.J., Le Maitre, R.W., Streckeisen, A., \& Zanettin, B. 1986, A chemical classification of volcanic rocks based on the total Alkali-Silica diagram. Journal of Petrology, 27(3): 745-750. doi:10.1093/petrology/27.3.745

Le Maitre, R.W.E., Bateman, P., Dudek, A., Keller, J., Lameyre, J., La Bas, M.J., Sabine, P.A., Schmid, R., Sorensen, H., Streckeisen, A.L., Woolley, A.R., \& Zanettin, B. 1989, A Classification of Igneous Rocks and Glossary of Terms; Recommendations of the International Union of Geological Science, Subcommission on the Systematics of Igneous Rocks. Blackwell Scientific Publications, Oxford, 193 p.

McDonough, W.F., \& Sun, S.-S. 1995, The composition of the Earth. Chemical Geology, 120: 223-253. doi:10.1016/0009-2541(94)00140-4

Philip, G., \& Williams-Thorpe, O. 1993, A provenance study of Jordanian basalt vessels of the Chalcolithic and Early Bronze Age I periods. Paléorient, 19(2): 51-63. doi:10.3406/paleo.1993.4596

Philip, G., \& Williams-Thorpe, O. 2000, The production and distribution of ground stone artefacts in the Southern Levant during the 5th-4th millennia BC: some implications of geochemical and petrographic analysis. Presented at Congress on the Archaeology of the Ancient Near East, Rome, 18-23 May 1998.

Philip, G., \& Williams-Thorpe, O. 2001, The production and consumption of basalt artefacts in the southern Levant during the 5th-4th millennia BC: a geochemical and petrographic investigation. In: Archaeological Sciences '97 - Proceedings of the Conference Held at the University of Durham, $2^{\text {nd }}-4^{\text {th }}$ September 1997 (Millard. A, Ed.), BAR International Series Vol. 939, Oxford, Archaeopress: p. 11-30.

Rosenberg, D., Shimelmitz, R., \& Nativ, A. 2008, Basalt bifacials production in the southern Levant: a glance at the quarry and workshop site of Giv ${ }^{\circ}$ at Kipod, Israel. Antiquity, 82(316): 367-376. doi:10.1017/s0003598x00096861

Rosenberg, D., \& Gluhak, T. 2016. Trade me an axe? Interpretive challenges of the distribution and provenance of Neolithic basaltic bifacial tools in Israel. Antiquity, 90(349): 48-63. doi: 10.15184/aqy.2015.191 
Rowan, Y.M. 1998, Ancient Distribution and Deposition of Prestige Objects: Basalt Vessels during Late Prehistory in the Southern Levant. Unpublished Ph.D dissertation, University of Texas, Austin, Texas. Pp. 518.

Rutter, G.P. 2003, Basaltic-Rock Procurement Systems in the Southern Levant: Case Studies from the Chalcolithic-Early Bronze I and the Late Bronze-Iron Ages. Unpublished Ph.D dissertation, University of Durham, Durham, 400 p.

Rutter, G., Pearson, D.G., Philip, G., Day, J.M.D., \& Ottley, C.J. 2003, The use of ICP-MS in provenancing igneous stone artefacts: examples from the southern Levant. In: Plasma Source Mass Spectrometry: Applications and Emerging Technologies (Holland, G., and Tanner, S.D., Eds.), The Royal Society of Chemistry, Cambridge: p. 209-219. doi:10.1039/9781847551689-00209

Rutter, G., \& Philip, G. 2008, Beyond provenance analyses: the movement of basaltic artefacts through a social landscape. In: New Approaches to Old Stones: Recent Studies of Ground Stone Artefacts (Rowan, Y.M., \& Ebeling, J.R., Eds.), Equinox Publishing, London: p. 343-358.

Schattner, U., \& Weinberger, R. 2008, A mid-Pleistocene deformation transition in the Hula basin, northern Israel: Implications for the tectonic evolution of the Dead Sea Fault. $G^{3}$ Geochemistry, Geophysics, Geosystems, 9(7). Accessed at: 10 July 2008. doi: 10.1029/2007gc001937

Shimelmitz, R., \& Rosenberg, D. 2016. The organization of basanite bifacial production at Giv’at Kipod Quarry, Israel: Towards an 'Alyawara Day’ model of extraction. Journal of Lithic Studies, 3(3): 23 p. doi:10.2218/jls.v3i3.1643

Sparks, R. T. 2007. Stone Vessels in the Levant. Palestine Exploration Found Annual VIII. Maney Publishing, Leeds, 488 p.

Stocks, D. A. 1993. Making stone vessels in ancient Mesopotamia and Egypt. Antiquity, 67(256): 596-603. doi: 10.1017/s0003598x00045804

Stocks, D. A. 1999. Stone sarcophagus in Ancient Egypt. Antiquity, 73(282): 918-922. doi:10.1017/S0003598X00065704

Stocks, D.A. 2003. Experiments in Egyptian Archaeology Stoneworking Technology in Ancient Egypt. Routledge, New York, 296 p.

Sneh, A., \& Weinberger, R. 2006, Rosh Pina, Sheet 2-IV, Geological Map of Israel 1:50,000, State of Israel, Ministry of National Infrastructures, Ministry of National Infrastructures, Geological Survey of Israel, Jerusalem.

Watts, B.G., Bennet III, M.E., Kopp, O.C. \& Mattingly, G.L. 2004, Geochemistry and petrography of basalt grindstones from the Karak Plateau, Central Jordan. Geoarchaeology, 19(1): 47-69. doi:10.1002/gea.10103

Williams-Thorpe, O., \& Thorpe, R. 1993, Geochemistry and trade of eastern mediterranean millstones from the Neolithic to Roman periods. Journal of Archaeological Science, 20(3): 263-320. doi: 10.1006/jasc.1993.1018

Xenophontos, C.; Elliott, C. \& Malpas, J. G. 1988. Major and trace-element geochemistry used in tracing the provenance of Late Bronze Age and Roman basalt artifacts from Cyprus. Levant, 20(1):169-183. doi:10.1179/lev.1988.20.1.169 